\title{
Correlation of CD133 and SOX2 Expression with Regional Lymph Nodes Metastatic Status in Invasive Breast Carcinoma of No Special Type
}

\author{
Sutrisno, Dyah Fauziah*, Gondo Mastutik \\ Department of Anatomical Pathology, Faculty of Medicine, Universitas Airlangga - Dr. Soetomo Hospital, Surabaya, Indonesia
}

\section{ARTICLE INFO}

Received : 01 July 2020

Reviewed : 03 August 2020

Accepted : 04 January 2021

Keywords:

breast cancer, invasive breast carcinoma of NST, CD133, SOX2, regional LN metastasis

*Corresponding author:

Dyah Fauziah

Department of Anatomical Pathology,

Faculty of Medicine, Universitas

Airlangga - Dr. Soetomo Hospital,

Surabaya, Indonesia

dyahf73@gmail.com

\author{
ABSTRACT
}

\begin{abstract}
Background: CD133 overexpression can increase cell proliferation, migration, and epithelialmesenchymal transition that promotes metastasis. CD133 expression is induced by hypoxiainduced factor (HIF) which requires SOX2 binding in the promoter region. SOX2 is an embryonal transcription factor that plays a role in the development of malignancy. The study aimed to analyze the expression of CD133 and SOX2 with regional lymph nodes (LN) metastatic status in invasive breast carcinoma of no special type (NST).
\end{abstract}

\begin{abstract}
Methods: The study was a cross-sectional design. Forty-five samples were retrieved from pathology archives in Dr. Soetomo Hospital from January to December 2018. Samples were divided into 2 groups, with and without regional LN metastasis. Immunohistochemistry with CD133 and SOX2 was applied to all samples. CD133 expression was assessed by immunoreactive score, and SOX 2 expression was assessed by the percentage of tumor positive cells.
\end{abstract}

\begin{abstract}
Results: There was no significant difference in CD 133 expression between invasive breast carcinoma of NST with and without regional LN metastases $(P=.990)$. A positive correlation was found in SOX2 expression between breast carcinoma with and without regional LN metastasis $\left(P=.000 ; r_{s}=.518\right)$. There was no correlation between CD133 and SOX2 expression $(P=.082)$, which means that the high expression of CD133 did not affect SOX2 expression.
\end{abstract}

Conclusions: CD133 expression was not significantly different in breast carcinoma with and without LN metastasis. The high expression of SOX2 was found significantly correlated with regional LN metastasis. SOX2 expression may become a potential prognostic marker in invasive breast carcinoma of NST.

\section{INTRODUCTION}

Breast cancer is the most common malignancy in women with an estimated 2.4 million new cases and a mortality rate of 523,000 per year in 2015 [1]. Globocan data for 2018 showed that breast cancer is the second most cancer worldwide after lung cancer, with a total number of 2,088,849 (11.6\%), and the fifthlargest mortality rate in the world at 626,679 people (6.6\%) [2]. Basic health research data issued by the Ministry of Health of the Republic of Indonesia in 2013 stated that breast cancer is second-highest cancer in women after cervical cancer with a prevalence of 0.5 $\%$ and an estimated number of patients as many as 61,682 people [3]. Tumor metastasis is one of the important factors which indicates the patient's prognosis, where $90 \%$ of the patient's death is caused by tumor with metastasis [4]. Several studies reported that cancer stem cells have an important role in the initiation and metastasis of cancer. One of the cancer stem cell markers is CD133 [5].

CD133 or Prominin-1 is a transmembrane glycoprotein consisting of 5 segments. As a marker of cancer stem cells, CD133 is first expressed in stem cells/hematopoietic progenitor cells [6]. CD133 was also detected in cancer stem cells of various solid tumors, such as brain tumors, colon, pancreas, prostate, lung, and liver [7]. Han et al. [5] in their study concluded that CD133 expression in breast cancer is associated with higher grade, lymph node metastases, negative ER / PR, positive HER2, increased staging, and poor survival rate. CD133 expression is induced by hypoxia-induced factor (HIF) and requires binding with sex-determining region $Y$ box 2 (SOX2) and OCT4 in the P1 promoter region, but SOX2 and OCT4 can increase CD133 transcription without the involvement of HIF [8]. 
SOX2 plays an important role in increasing the expression of CD133 [9]. SOX2 is a transcription factor associated with pluripotency in embryonal stem cells. SOX2 has an important role in the process of organogenesis and the embryonal development of several tissues. A high SOX2 expression is associated with a low level of differentiation. The healthy adult breast tissue does not show significant expression of SOX2, but SOX2 is detected in some patients with breast cancer. This suggests that SOX2 activation plays an important role in the development of malignancy [10]. Increased SOX2 expression in breast carcinoma also induces epithelial-mesenchymal transitions (EMT) by activating the $\mathrm{Wnt} / \beta$-catenin pathway which will increase the potency of tumor metastasis [11].

To date, published studies of both CD133 and SOX2 expressions related to regional LN metastasis in invasive breast carcinoma are limited. This study was conducted to analyze the correlation between CD133 and SOX2 expressions with the metastatic status of regional LN in invasive breast carcinoma of NST.

\section{METHODS}

The study was an observational analytic study with a cross-sectional approach. Samples were obtained from the archives in the Laboratory of Anatomical Pathology, Dr. Soetomo Hospital, Surabaya, from January until December 2018, which met the inclusion criteria. The latter was invasive breast carcinoma of NST from mastectomy and regional lymph node dissection procedure which contained at least 6 LN. The Sample size was calculated using a formula, and a minimum of 20 samples per group was obtained. Forty-five samples of invasive breast carcinoma of NST were included. The samples were divided into 2 groups, consisting of 23 cases of breast cancer with regional LN metastasis and 22 cases of breast cancer without regional LN metastasis. Both groups had tumor size from T1 - T4 according to T stages. Ethical approval was obtained from the ethical and health research committee of Dr. Soetomo Hospital with the registration number of 1659/KEPK/XI/2019.

Immunohistochemistry with CD133 monoclonal antibody (dilution 1: 1000, Abcam) and SOX2 monoclonal antibody (dilution 1: 100, Biocare) were applied on all samples. CD133 is expressed in the membrane and or cytoplasm of tumor cells. CD133 expression was evaluated using immunoreactive score (IRS) by assessing the percentage and intensity of tumor positive cells. The percentage of positive cells was scored as 0 (no positive cells), 1 (positive cells $<10 \%$ ), 2 (positive cells $10-50 \%$ ), 3 (positive cells $51-80 \%$ ), and 4 (positive cells
$>80 \%$ ). The intensity was scored as 0 (negative), 1 (mild), 2 (moderate), and 3 (strong). IRS was calculated by multiplying the percentage and intensity. Final scores were categorized as $0-1=$ negative, $2-4=$ weak expression, $6-8=$ moderate expression, and $9-12=$ strong expression [12]. SOX2 is expressed in the nucleus of tumor cells. The percentage of positive cells was assessed as score $0=$ no positive cells, $1=$ positive on $<10 \%$ tumor cells, 2 = positive on $10 \%-50 \%$ tumor cells, and $3=$ positive on $>50 \%$ of tumor cells [10].

The data obtained were processed using the IBM SPSS 25.0 software for Windows 10.0. The statistical analysis of the Mann-Whitney test was performed to evaluate the difference in the expressions of CD133 and SOX2 $(P<.05)$. The correlation between CD133 and SOX2 expressions with regional LN metastatic status in invasive breast carcinoma of NST was tested using the Spearman correlation test $(P<.05)$.

\section{RESULTS}

All samples were female with an age range of 32 to 69 years, and the average age of the samples was $51.98 \pm 10.8$ years. The characteristics of the samples are described in Table 1 . There was a statistical correlation between age groups and $L N$ status $(P=.017)$. The grading of invasive breast carcinoma was determined using modified Bloom and Richardson criteria, based on tubular formation, nuclear pleomorphism, and mitotic count. Most research samples were in grade 3 . There was no significant correlation between tumor grade and LN status $(P=.226)$. The tumor size was categorized using the 8th edition of the TNM American Joint Committee on Cancer (AJCC) stage. The statistical analysis showed a significant correlation between tumor size and $\mathrm{LN}$ status $(P=.015)$.

The expression of CD133 in carcinoma cells is depicted in Figure 1. The IRS of CD133 for both groups is described in Table 1. Mann-Whitney test showed no difference of CD133 expression in invasive breast carcinoma of NST with and without regional LN metastases $(P=.990)$. Spearman correlation test showed no significant correlation between CD133 expressions with regional LN metastatic status $(P=.990)$. These results indicated that the level of CD133 expression had no association with the regional $\mathrm{LN}$ metastasis $(P>.05)$ (Figure 3A).

The expression of SOX2 in carcinoma cells is depicted in Figure 2. The scores of SOX2 expression for both groups are described in Table 1 . Mann-Whitney test showed a significant difference in SOX2 expression in invasive breast carcinoma with and without regional LN metastasis $(P=.001)$. The Spearman correlation test 
showed a significant relationship between SOX2 expression with regional LN metastatic status with a positive correlation coefficient value $r s=.518(P=.000)$ (Figure 3B). These results indicated that the higher SOX2 expression means the higher potency for regional LN metastasis $(P<.05)$.

Multivariate analysis with logistic regression, including age, tumor size, and SOX2 expression as variables, showed that only SOX2 expression retained a significant correlation with regional $\mathrm{LN}$ metastasis $(P$ $=.007$ ) (Table 2).

The Spearman statistical test showed no significant correlation between CD133 and SOX2 expressions ( $P=$ .082) (Figure $3 C$ ). This indicated that $C D 133$ expression had no influence on SOX2 expression in invasive breast carcinoma with and without regional LN metastases.
Table 1. Characteristics of research samples

\begin{tabular}{|c|c|c|c|c|}
\hline Characteristics & Metastasis & Non-Metastasis & Total & $P$ \\
\hline \multicolumn{5}{|l|}{ Age (y.o) } \\
\hline $31-40$ & 3 (13.0\%) & 7 (31.8\%) & $10(22.2 \%)$ & $.017^{\mathrm{c}}$ \\
\hline $41-50$ & $3(13.0 \%)$ & $5(22.7 \%)$ & $8(17.8 \%)$ & \\
\hline $51-60$ & 7 (30.4\%) & 7 (31.8\%) & $14(31.1 \%)$ & \\
\hline $61-70$ & $10(43.5 \%)$ & $3(13.6 \%)$ & $13(28.9 \%)$ & \\
\hline \multicolumn{5}{|l|}{ Grading } \\
\hline Grade 1 & $1(4.3 \%)$ & $3(13.6 \%)$ & 4 (8.9\%) & .226 \\
\hline Grade 2 & $2(8.7 \%)$ & $3(13.6 \%)$ & 5 (11.1\%) & \\
\hline Grade 3 & $20(87.0 \%)$ & $16(72.7 \%)$ & $36(80.0 \%)$ & \\
\hline \multicolumn{5}{|l|}{ Tumor size } \\
\hline $\mathrm{T} 1$ & $2(8.7 \%)$ & $3(13.6 \%)$ & $5(11.1 \%)$ & $.015^{c}$ \\
\hline $\mathrm{T} 2$ & 7 (30.4\%) & $13(59.1 \%)$ & $20(44.4 \%)$ & \\
\hline T3 & $6(26.1 \%)$ & $5(22.7 \%)$ & $11(24.4 \%)$ & \\
\hline $\mathrm{T} 4$ & $8(34.8 \%)$ & $1(4.6 \%)$ & $9(20.0 \%)$ & \\
\hline \multicolumn{5}{|l|}{ Estrogen receptor ${ }^{a}$} \\
\hline Positive & $19(82.6 \%)$ & $13(61.9 \%)$ & 32 (72.7\%) & .124 \\
\hline Negative & $4(17.4 \%)$ & $8(38.1)$ & $12(27.3 \%)$ & \\
\hline \multicolumn{5}{|l|}{ Molecular subtype ${ }^{b}$} \\
\hline Luminal A & $1(4.4 \%)$ & 2 (9.1\%) & $3(6.7 \%)$ & .441 \\
\hline Luminal B & $14(60.9 \%)$ & $8(36.4 \%)$ & $22(48.9 \%)$ & \\
\hline HER2-enriched & $3(13.0 \%)$ & 2 (9.1\%) & 5 (11.1\%) & \\
\hline Triple-negative & $0(0.0 \%)$ & $6(27.3 \%)$ & $6(13.3 \%)$ & \\
\hline NA & $5(21.7 \%)$ & $4(18.2 \%)$ & $9(20.0 \%)$ & \\
\hline \multicolumn{5}{|l|}{ CD133 expression } \\
\hline Negative & $0(0.0 \%)$ & $0(0.0 \%)$ & $0(0.0 \%)$ & .990 \\
\hline Weak & $2(8.7 \%)$ & $3(13.6 \%)$ & $5(11.1 \%)$ & \\
\hline Moderate & $8(34.8 \%)$ & $6(27.3 \%)$ & $14(31.1 \%)$ & \\
\hline Strong & $13(56.5 \%)$ & $13(59.1 \%)$ & $26(57.8 \%)$ & \\
\hline \multicolumn{5}{|l|}{ SOX2 expression } \\
\hline Negative & 9 (39.1\%) & $20(90.9 \%)$ & $29(64.4 \%)$ & .000 \\
\hline Score 1 & $10(43.5 \%)$ & $1(4.5 \%)$ & $11(24.4 \%)$ & \\
\hline Score 2 & 4 (17.4\%) & $1(4.5 \%)$ & $5(11.1)$ & \\
\hline Score 3 & $0(0.0 \%)$ & $0(0.00 \%)$ & $0(0.0 \%)$ & \\
\hline
\end{tabular}

$a=1$ non-metastasis sample did not have immunohistochemical staining

$b=$ immunohistochemical staining were not conclusive due to equivocal HER2 or did not have any Ki67 $\mathrm{c}=$ statistically significant $(P<.05)$ 


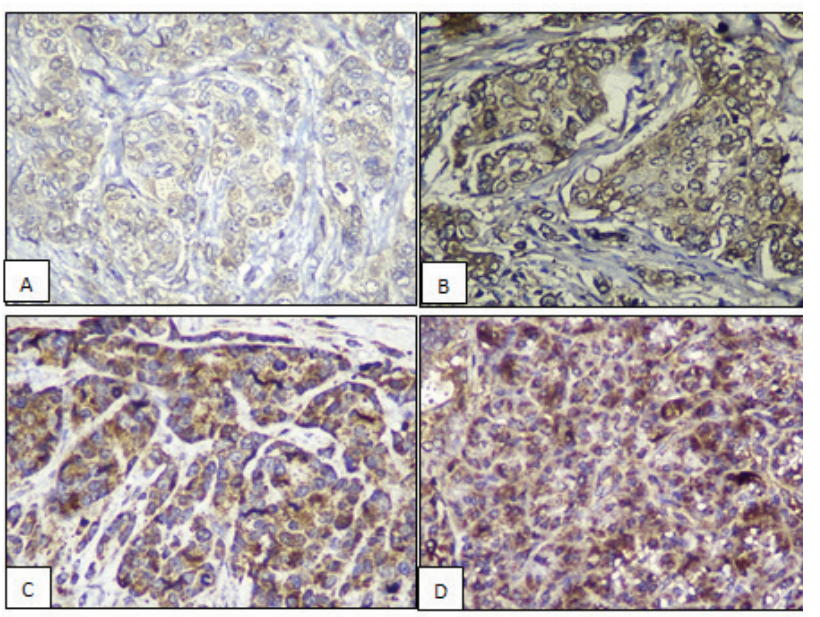

Figure 1. CD133 expression in the membrane and cytoplasm of tumor cells. (A) weak intensity, (B) moderate intensity, (C) strong intensity, (D) positive control with pancreatic tissue (all in 400X magnification).

Table 2. Variables in the equation of multivariate analysis

\begin{tabular}{lllll}
\hline \multirow{2}{*}{ Variables } & Sig. & Exp(B) & \multicolumn{2}{c}{$95 \%$ C.I for EXP(B) } \\
\cline { 5 - 6 } & & & Lower & Upper \\
\hline Last Step & & & & \\
SOX2 & 0.007 & & & \\
SOX2 (1) & 0.066 & 0.113 & 0.011 & 1.154 \\
SOX2 (2) & 0.550 & 2.500 & 0.124 & 50.444
\end{tabular}

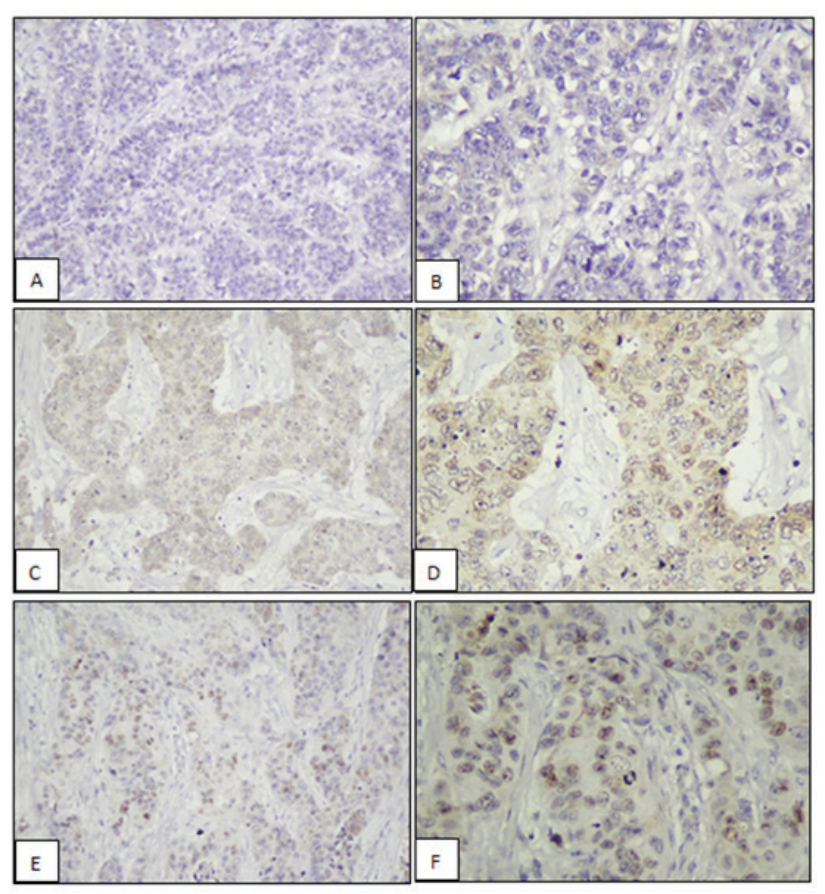

Figure 2. SOX2 expression in the nucleus of tumor cells. (A) and (B) show negative scores (magnification of 200x and 400x). (C) and (D) show score 1 with a percentage $<10 \%$ (magnification of 200x and 400x). (E) and (F) show score 2 with percentages $>10 \%$ and $<50 \%$ (magnification of $200 x$ and $400 x$ ).

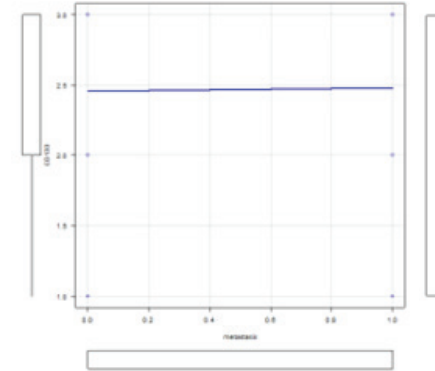

(A)

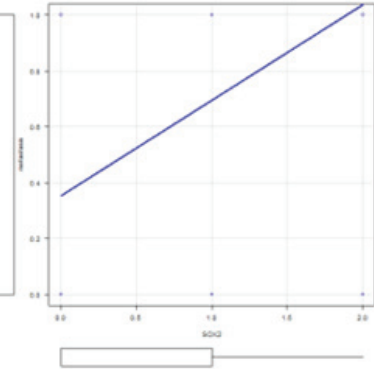

(B)

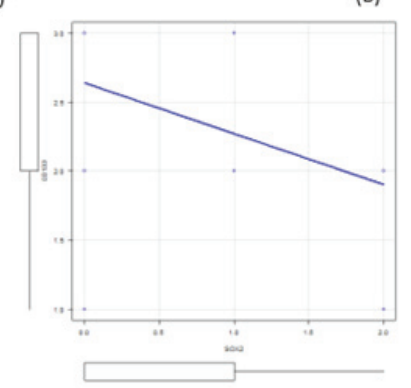

(C)

Figure 3. Spearman correlation graphic. (A) Correlation between CD133 expression and regional LN metastasis. (B) Correlation between SOX2 expression and regional LN metastasis. (C) Correlation between $\mathrm{CD} 133$ and SOX2 expression with regional LN metastasis.

\section{DISCUSSION}

CD133 is a pentaspan-transmembrane glycoprotein with molecular weights of around 115-120 kDa and contains 850-865 amino acids. CD133 consists of extracellular $\mathrm{N}$-terminals and 5 domains with 2 large extracellular-loops, 2 small intracellular-loops, and an intracellular C-terminal domain. The gene that encodes CD133 is located on chromosome 4 and contains at least 37 exons. This glycoprotein is called prominin because of its location in plasma membrane protrusion $[13,14]$. CD133 or prominin-1 is initially associated with cholesterol called a lipid raft and later described as a specific biomarker for hematopoietic progenitor cells. CD133 is known as an important biomarker for identifying and isolating cancer stem cells (CSC) in several malignancies, including breast carcinoma [15]. Several studies have shown that CD133 is an important biomarker in several normal tissues such as the brain, kidney, prostate, bone marrow, liver, pancreas, and skin, as well as in solid tumor tissue such as brain tumors, colon, pancreas, prostate, lung, and liver $[7,13]$. CD133+ cells have stemness properties, such as drug resistance, self-renewal, differentiation, and high proliferation abilities, and they can form tumors in xenograft [15].

The meta-analysis research conducted by Han et al. [5] of 11 previous studies included 7 studies in Asia, 2 studies in Europe, 1 study in Oceania, and 1 study in Africa and involved 1,447 patients. The study reported 
that CD133 expression in breast cancer was closely related to high cancer grading $(P<.001)$, LN metastatic status $(P<.001)$, higher stage of cancer $(P<.001)$, negative ER expression $(P=.018)$, negative $\mathrm{PR}$ expression $(P<.001)$, positive HER2 expression $(P=.039)$, and worse overall survival $(P<.001)[5]$. Research by Zhao et al. [16] in triple-negative breast cancer showed that CD133 expression was related to tumor size $(P=.022)$, clinical-stage $(P=.001)$, and regional LN metastasis $(P=.001)$. CD133 expression also reflected survival rates, where patients with a positive CD133 showed a lower average survival rate of 34 months compared to patients with a negative CD133 of 52.3 months $(P=.0001)$ [16].

The result of this study showed no significant difference in CD133 expression in invasive breast carcinoma with and without regional LN metastasis. The insignificant results may be because in this study the majority of samples had grade 3 , so both groups showed equally strong CD133 expression. Research by Abdelrahman et al. [17] in astrocytic glioma showed that CD133 expression was significantly related to the grade and size of the tumor. Xiao et al. [18] in meningiomas stated that CD133 expression was associated with higher tumor grade. CD133 expression in esophageal squamous cell carcinoma also showed a linear relationship with LN metastasis, depth of invasion, tumor grade, and TNM stage, as well as the results of meta-analysis research conducted by Han et al. [5] who reported that CD133 expression in breast cancer was closely related to high cancer grading $[5,19]$. Another possibility of this unproven study might be due to the Src-FAK factor which is not activated. A literature study states that CD133 affects the migration of cancer cells by the interaction between cells or cells with the extracellular matrix. Research by Li et al. [20] and Jang et al. [21] reported that CD133 could activate the SrcFAK pathway. CD133 interacts with $\mathrm{Src}$ in the amino acid region 845 - 857 C-terminal domain. Tyrosine-828 and tyrosine- 852 cells in the cytoplasmic-tail of CD133 are phosphorylated by the Src kinase family which then activates FAK and increases migration. FAK is a kinase substrate of Src that is important in mediating tumor development and metastasis. Src-FAK complex plays an important role in the regulation of adhesion and cancer cell invasion ability.

Sex-determining region $Y$ - box 2 (SOX2) is a single exon gene without introns located on chromosome $3 q 26.3$ - q27, encoding 317 amino acids, and is a member of the SOX transcription factor family that regulates mammalian development. SOX2 has a key role in maintaining self-renewal and pluripotential properties in undifferentiated embryonic stem cells and in maintaining the character of embryonal and neuronal stem cells [23]. SOX2 is expressed in early development and is very important for the maintenance of pluripotent stem cell populations. SOX2 expression changes in several types of tumors with varying degrees of differentiation, consistent with its function in maintaining the properties of stem cells in embryonal cells. Several studies showed that this gene plays an important role in forming the undifferentiated stem cell phenotype characteristics associated with basal-like breast carcinoma subtype [11]. Recent studies have shown that SOX2 is overexpressed in early breast carcinoma and has a positive correlation with tumor size [10]. SOX2 is more often expressed in basal-like and triple-negative breast carcinoma subtypes. It increases cell proliferation and metastasis and is associated with shorter survival rates [11].

The results of this study are in line with several previous SOX2-related studies. Research by Neumann et al. [24] mentioned that the increased expression of SOX2 protein was significantly correlated with LN metastasis $(P=.006)$, distant metastasis $(P=.022)$, and tumor grade $(P=.043)$ in colon cancer but did not correlate with tumor size, sex, and age of the patient. Research by Gao et al. [25] in esophageal squamous cell carcinoma showed that overexpression of SOX2 increased migration and invasion of cancer cells. Research by Liu et al. [26] in squamous cell carcinoma of the tongue showed that SOX2 overexpression was related to tumor size $(P=.035)$, cell differentiation $(P=.042)$, and LN metastasis $(P=.018)$.

Research by Wang and Xie [27] on the role of SOX2 in breast cancer angiogenesis reported that SOX2 has a significant relationship with regional LN metastases $(P<.01)$ and clinical stage $(P<.05)$. Study by Lengerke et al. [10] in early-stage breast cancer mentioned a significant relationship between SOX2 expression and LN metastasis $(P=.018)$ and tumor size $(P=.047)$. Liu et al. [28] conducted a study of SOX2 expression in 237 patients with triple-negative breast cancer. The study found that SOX2 expression was associated with regional LN metastases $(P<.001)$, tumor size $(P<.001)$, and clinical stage $(P<.001)$. The research was continued by making a breast cancer xenograft model in mice to find out the role of SOX2 further. The results showed that SOX2 inhibition reduced tumor cell proliferation, reduced tumor cell invasion, and increased tumor cell apoptosis in triple-negative breast cancer. SOX2 inhibition also reduced tumorigenesis and metastasis.

Research by Li et al. [4] in breast cancer mentioned the relationship between SOX2 expression and tumor grade $(P=.005)$, TNM stage $(P=.002)$, and regional LN metastases $(P<.001)$. The study was continued with a tumor model in mice. It was found that SOX2 increased tumor cell migration and metastasis. The process of metastasis was further investigated. Their study found that SOX2 could improve the EMT process, induced by TGF- $\beta$. The increase in EMT by SOX 2 was also through the $\beta$-catenin signal. EMT is known as a central mechanism in the invasion and metastasis of 
cancer by loss of connection between epithelial cells and the ability to migrate. Overexpression of SOX2 triggers EMT characterized by decreased epithelial markers such as E-cadherin and increased mesenchymal markers such as $\mathrm{N}$-cadherin, vimentin, fibronectin, and $\alpha$-SMA. Research by Gao et al. [25] reported that this increase of EMT activity was mediated by Slug, which is a transcription factor that is activated during the EMT process. The increased Slug expression is directly related to the increase in tumor grade, metastasis, and poor prognosis in various malignancies $[25,26]$.

Spearman correlation test between CD133 expression and SOX2 expression on regional LN metastatic status showed that there was no significant relationship between CD133 and SOX2 expressions $(P>.05)$. This result was different from the study of Abdelrahman et al. [17] on astrocytic glioma which stated that there was a significant relationship between CD133 and SOX2 expressions, where $63.6 \%$ of low SOX2 cases had CD133 focal expression, and $94.1 \%$ of high SOX2 cases had a diffuse expression of CD133 $(P<.001)$. Research by Saigusa et al. [29] on colorectal cancer stated that postchemoradiotherapy patients showed a positive relationship between CD133 and SOX2 with a correlation coefficient $r_{s}=.89(P<.0001)$. Their research also showed that the expression of CD133 and SOX2 after chemoradiotherapy was associated with recurrence and decreased disease-free survival.

A high SOX2 expression should be followed by an increase in CD133 expression. Research by Lida et al. [8] on lung cancer cells proved that CD133 expression under hypoxic conditions would be induced by HIF and required binding with OCT4 and SOX 2 in the $\mathrm{P} 1$ promoter region. The study used the immunoprecipitation method to prove how big the role of OCT4 and SOX2 is in activating CD133 expression. The results showed that increased OCT4 and SOX2 expression could also increase CD133 transcription even in normoxic conditions and without HIF involvement. The study also confirmed that the inhibition of OCT4 in P1 promoters reduced CD133 expression by one-third while SOX2 inhibition in P1 promoters reduced CD133 expression by half.

The CD133 expression level was not in line with SOX2 expression in this study. The most likely explanation was the presence of other inductors besides OCT4 and SOX2 in activating CD133 expression. Research by Konishi et al. [30] in gastric cancer reported that Notch-1 could directly induce CD133 expression in diffuse-type gastric cancer. An increase in Notch-1 was followed by an increase in CD133 expression; conversely, inhibition of the Notch-1 signal was followed by a decrease in CD133 expression. Further research using the chromatin immunoprecipitation method showed that the Notchintracellular domain (NICD) bound to the RBP-Jk site at promoter 5 of CD133. This bond triggered an increase in CD133 transcription so that CD133 expression increased.

This present study was conducted in a tertiary referral hospital; hence, most breast cancer patients come with advanced-stage and high-grade tumors. This may limit the study due to the limited number of cases in early and low grades.

\section{CONCLUSIONS}

CD133 expression was not significantly different in breast carcinoma with and without LN metastasis. The high expression of SOX2 was found significantly correlated with regional LN metastasis. SOX2 expression may become a potential prognostic marker in invasive breast carcinoma of NST.

\section{DECLARATIONS}

\section{Competing of Interest}

The authors declare no competing interest in this study.

\section{Acknowledgement}

The authors wish to thank Dr. Budi Utomo, Department of Public Health and Preventive Medicine, Universitas Airlangga for statistical analysis.

\section{REFERENCES}

1. Bellanger $\mathrm{M}$, Zeinomar $\mathrm{N}$, Tehranifar $\mathrm{P}$, Terry MB. Are global breast cancer incidence and mortality patterns related to country spesific economic development and prevention strategies?. J Glob Oncol. 2018;4:1-16.

2. GLOBOCAN. International Agency for Research on Cancer. World Health Organization. 2018.

3. Kementerian Kesehatan RI. Kanker payudara. Infodatin Pusat Data dan Informasi Kementerian Kesehatan RI. 2016.

4. Li X, Xu Y, Chen $Y$, et al. SOX2 promotes tumor metastasis by stimulating epithelial-to-mesenchymal transition via regulation of WNT/ß-catenin signal network. Cancer Lett. 2013;336(2):379-89.

5. Han L, Gao X, Gu X, et al. Prognostic significance of cancer stem cell marker CD133 expression in breast cancer. Int J Clin Exp Med. 2017;10(3):4829-37.

6. Li Z. CD133: a stem cell biomarker and beyond. Exp Hematol Oncol. 2013;2(1):17. 
7. Glumac PM, Lebeau AM. The role of CD133 in cancer: a concise review. Clin Transl Med. 2018;7:18.

8. Lida H, Suzuki M, Goitsuka R, Ueno H. Hypoxia induces CD133 expression in human lung cancer cells by up-regulation of OCT3/4 and SOX2. Int J Oncol. 2012;40:71-9.

9. Liou G. CD133 as a regulator of cancer metastasis through the cancer stem cells. Int J Biochem Cell Biol. 2019;106:1-7.

10. Lengerke C, Fehm T, Kurth R, et al. Expression of the embryonic stem cell marker SOX2 in early-stage breast carcinoma. BMC Cancer. 2011;11:42.

11. Mehta GA, Khanna P, Gatza ML. Emerging role of SOX2 proteins in breast cancer development and maintenance. J Mammary Gland Biol Neoplasia. 2019;24(3):213-30.

12. Bock C, Kuhn C, Ditsch N, et al. Strong correlation between $\mathrm{N}$-cadherin and $\mathrm{CD} 133$ in breast cancer: role of both markers in metastatic events. J Cancer Res Clin Oncol. 2014;140:1873-81.

13. Behrooz AB, Syahir A, Ahmad S. CD133: beyond a cancer stem cell biomarker. Journal of Drug Targeting. 2019;27(3):257-69.

14. Aghajani M, Mansoori B, Mohammadi A, et al. New emerging roles of $\mathrm{CD} 133$ in cancer stem cell: signaling pathway and miRNA regulation. Cell Physiol. 2019;234(12):21642-61.

15. Tume L, Paco K, Ubidia-Incio R, Moya J. CD133 in breast cancer cells and in breast cancer stem cells as another target for immunotherapy. Gaceta Mexicana De Oncologia. 2016;15(1):22-30.

16. Zhao $P$, Lu Y, Jiang $X$, Li X., Clinicopathological significance and prognostic value of CD133 expression in triple-negative breast carcinoma. Cancer Sci. 2011;102(5):1107-11.

17. Abdelrahman $A E$, Ibrahim $H M$, Elsebai $E A$, et al. The clinicopathological significance of $\mathrm{CD} 133$ and SOX2 in astrocytic glioma. Cancer Biomark. 2018;23:391-403.

18. Xiao Z-Y, Chen X-J, Pan Q, et al. Expression of nestin, CD133 and SOX2 in meningiomas. Turk Neurosurg. 2018;28(6):910-14.
19. Zhao W, Qin Y, Wu X, et al. Correlation of CD133, E-cadherin, and $ß$-catenin with metastasis and prognosis in esophageal squamous cell carcinoma. Int J Clin Exp Med. 2018;11(9):8583-9592.

20. Li C, Li $Y$, Xing $Y$, et al. The interaction between cancer stem cell marker CD133 and Src protein promotes focal adhesion kinase (FAK) phosphorylation and cell migration. The J Biol Chem. 2016;291(30):15540-50.

21. Jang J, Song Y, Kim S, et al. Potential mechanism of CD133 in cancer stem cells. Life Sci. 2017;184:25-29.

22. Aghajani M, Mansoori B, Mohammadi A, et al. New emerging roles of CD133 in cancer stem cell: signaling pathway and miRNA regulation. J Cell Physiol. 2019;234(12):21642-61.

23. Mansouri S, Nejad R, Karabork $M$, et al. SOX2: regulation of expression and contribution to brain tumors. CNS Oncology. 2016;5(3):159-73.

24. Neumann J, Bahr F, Horst D, et al. SOX2 expression correlates with lymph-node metastasis and distant spread in right-sided colon cancer. BMC Cancer. 2011;11: 518.

25. Gao H, Teng C, Huang W, Peng J, Wang C. SOX2 promotes the epithelial to mesenchymal transition of esophageal squamous cells by modulating slug expression through the activation of STAT3/HIF- $\alpha$ signaling. Int J Mol Sci. 2015;16(9):21643-657.

26. Liu X, Qiao B, Zhao T, et al. SOX2 promotes tumor agressivenes and epithelial-mesenchymal transition in tongue squamous cell carcinoma. Int J Mol Med. 2018;42(3):1418-26.

27. Wang $\mathrm{H}$, Xie J. The role of SOX2 in angiogenesis in breast cancer. Int J Clin Exp Pathol. 2018;11(5):2805-10.

28. Liu P, Tang H, Song C, et al. SOX2 promotes cell proliferation and metastasis in triple negative breast cancer. Front Pharmacol. 2018;9:942.

29. Saigusa S, Tanaka K, Toiyama Y, et al. Correlation of CD133, OCT4, and SOX2 in rectal cancer and their association with distant recurrence after chemotherapy. Ann Surg Oncol. 2009;16(12):3488-98.

30. Konishi H, Asano N, Imatani A, et al. Notch1 directly induced CD133 expression in human diffuse type gastric cancers. Oncotarget. 2016;7(35):56598-607. 